\title{
Aptitud ecoturística en la sierra nevada de Texcoco, Estado de México
}

\section{Ecotourist flair in the sierra nevada of Texcoco, Mexico state}

\author{
Marcelino A. Pérez-Vivar', Manuel de J. González-Guillén', J. René Valdez-Lazalde², \\ Héctor M. de los Santos-Posadas² y Gregorio Ángeles-Pérez²
}

\begin{abstract}
1 Universidad Autónoma Chapingo. Chapingo, Edo. 2 Postgrado Forestal, Colegio de Posgraduados. México. marcelpv@colpos.mx Montecillo, Edo. México. manuelg(a)colpos.mx valdez@colpos.mx, hmsantos@colpos.mx, gangeles(a)colpos.mx.
\end{abstract}

\section{RESUMEN}

En el presente estudio se puso en operación un sistema de cómputo (software) desarrollado ad hoc para determinar la aptitud de áreas forestales para la realización de actividades ecoturísticas, dicho estudio se realizó en la Sierra Nevada de Texcoco, Estado de México. El sistema utiliza como insumos información sobre condiciones del ambiente relevantes para el desarrollo adecuado de actividades ecoturísticas, expresadas como una jerarquía de criterios, subcriterios y variables, así como información temática del área de evaluación. La información referente a las condiciones del ambiente se recolectó a través de una encuesta aplicada a instructores de niños exploradores, profesores de licenciatura y postgrado, estudiantes en el último año de licenciatura e integrantes de una asociación civil, todos ellos relacionados con el tema de ecoturismo. Con respecto a la información temática, se utilizaron mapas digitales bajo sistema de coordenadas UTM NAD27 correspondientes a la carta Chalco "E14B31", escala 1:50000 de INEGI. Como resultado, el sistema generó mapas de aptitud con valores en un gradiente de 0 a 1 para las actividades de campismo, caminata y día de campo, en donde el valor 1 indica la presencia de condiciones ideales para el desarrollo de la actividad y cero la ausencia total. Adicionalmente se evaluó el funcionamiento del sistema, mostrando un desempeño confiable y constatando que posee gran potencial para la representación de relaciones complejas, cualidades que le pueden llevar representar una herramienta valiosa, eficaz y eficiente como instrumento de apoyo al proceso de toma de decisiones y planificación del uso de la tierra.

PALABRAS CLAVE: SIG, evaluación multicriterio, ecoturismo.

\section{ABSTRACT}

In the present study a computer (software) system was developed in order to determine the potential of forest areas for performing ecotourist activities; such study was carried out in the Sierra Nevada of Texcoco, State of Mexico. The system utilizes as input information about conditions of the environment, important for adequate development of ecotourism activities, expressed as rank of criteria, sub-criteria, and variables, as well as subject matter information of the area of evaluation. The information, regarding the conditions of environment, was gathered by means of survey, applied to instructors of scout children, professors of university and postgraduate degree, students in the last year of degree, and members of civil associations, related all of them to the topic of ecotourism. With respect to thematic information, digital maps were used under the system of UTM NAD27 coordinates, corresponding to the letter Chalco "E14B31", INEGI scale 1:50000. As a result, the system generated maps of capacity with values in a gradient from 0 to 1 for the activities of camping, long walk (hiking), and days out in the open, where value 1 indicates the presence of conditions, ideal for the development of the activity, and zero for its total absence. Additionally, the functioning of the system was evaluated demonstrating reliable performance and verifying of being endowed of potential for the representation of complex relations, qualities which may lead to stand for a valuable tool, efficacious and efficient as an instrument of support for the process of decision-taking and planning of land use.

KEY WORDS: GIS, multiple criteria evaluation, ecotourism. 


\section{INTRODUCCIÓN}

La importancia de la naturaleza en la existencia del ser humano es incuestionable. Existen alrededor de 30 funciones de interés para el hombre que tienen lugar en los ecosistemas (por ej., las áreas recreativas y facilidades para el turismo) (De Groot, 2006), lo que proporciona una serie de valores sociales que se constituyen en elemento fundamental para la salud física y mental (Gómez y De Groot, 2007).

El turismo ha crecido vertiginosamente impactando los ambientes en los que se desarrolla (Kimmel, 1999), masificándose al grado de amenazar los ambientes donde se practica (Pérez de las Heras, 2003). Por ello, el ecoturismo surge como alternativa que busca establecer una relación benéfica entre sociedad-naturaleza-comunidad local (Wunder, 1999); sin embargo, su desarrollo enfrenta el reto de conjuntar la visión de crecimiento sin límites con la visión de limitar el desarrollo imponiendo criterios de baja intensidad y mínimo impacto ecológico (Chávez, 2005), un reto que no siempre se alcanza. Por lo anterior, es vital que la planificación de una iniciativa de ecoturismo se desarrolle de acuerdo con la aptitud y capacidad de un ambiente (López y Triviño, 2004).

Han existido experiencias que abordan el estudio de la potencialidad ecoturística desde la perspectiva de la aptitud ${ }^{1}$ de los espacios para el desarrollo del ecoturismo. En ellas se observan diversidad de objetivos y metodologías específicas de tipo cualitativo y cuantitativo, siendo las últimas las de mayor confiabilidad (Pérez et al., 2012). Un elemento común en algunas metodologías de tipo cuantitativo son las herramientas en el ámbito de la "Toma de Decisiones"2; por ejemplo la Evaluación Multicriterio (EMC), la cual emplea técnicas como la Combinación Lineal Ponderada, Electre, Proceso Jerárquico Analítico (Saaty, 1990) y Promethee, en donde el uso de los Sistemas

\footnotetext{
I La aptitud del suelo está dirigida a la evaluación de los recursos naturales y la regulación de actividades humanas en un espacio (Bandyopadhyay et al., 2009) identificando la capacidad de una unidad de suelo para alojar un uso con base en requerimientos específicos (suelo, precipitación, temperatura, otros) y preferencias (Bojórquez et al., 1999).

2 "Serie de conceptos teóricos y articulaciones lógicas que pretenden explicar y predecir el comportamiento de los agentes tomadores de decisiones" (Romero, 1993)
}

de Información Geográfica (SIG) constituye un elemento común (Franco et al., 2009; Kenan, 2006; Blancas et al., 2009).

En su mayoría, las metodologías de tipo cuantitativo abordan la aptitud ecoturística evaluando condiciones físicas de un espacio y contrastándolas con aquellas requeridas para el desarrollo del ecoturismo entendido éste como "una modalidad turística que consiste en la visita a áreas naturales con poca perturbación para apreciar sus atractivos naturales así como cualquier manifestación cultural, en un proceso que promueve la conservación y que involucra a las comunidades locales" (Ceballos, 1998), sin hacer énfasis en que el ecoturismo contempla una diversidad de actividades con características específicas.

En la Sierra Nevada del municipio de Texcoco, han surgido iniciativas de proyectos de ecoturismo, con el afán de aprovechar en forma sustentable la belleza paisajística de sus áreas forestales (Caballero, 2007). Ello hace necesario contar con trabajos que determinen la aptitud ecoturística de los terrenos con base en la medición de variables del medio físico y la generación de un valor de compatibilidad entre éstas y un uso específico.

El presente trabajo constituye un estudio preliminar, que en una mecánica distinta a los trabajos realizados anteriormente, evalúa la aptitud ecoturística de una región estableciendo actividades ecoturísticas específicas y definiendo para ellas las condiciones biofísicas (cartografiables) del medio más apropiadas para su desarrollo. Posterior a ello se aplica un software que integra una metodología para la generación de valores de aptitud. El software se diseñó ad hoc para evaluar la aptitud ecoturística (Pérez et al., 2013; Pérez et al., 2011) mediante el desarrollo de un modelo conceptual que combina herramientas de evaluación multicriterio con operaciones SIG, y que contrasta los requerimientos ambientales necesarios para el desarrollo de una actividad ecoturística específica, con las características biofísicas del espacio disponible para generar valores de aptitud. La finalidad del proceso es la obtención de un mapa de aptitud que identifique las áreas más adecuadas para el desarrollo de cada actividad de interés. 
No se pretende que el trabajo sea exhaustivo, es un estudio exploratorio cuya relevancia radica en presentar una metodología para abordar la evaluación de la aptitud ecoturística. La información generada para efectuar la determinación de aptitud es solo una muestra del gran universo de vías para determinar los gustos y las preferencias en las cualidades que un espacio debe poseer para realizar una actividad específica. Se intenta que el estudio constituya una referencia para futuros y necesarios trabajos de investigación y que, en conjunto, puedan lograr una aproximación cada vez mayor al entendimiento del complejo problema que involucra la aptitud ecoturística.

\section{OBJETIVO}

Aplicar un sistema de cómputo (software) en el ámbito de los SIG para determinar la aptitud de zonas con potencial para el desarrollo de actividades ecoturísticas en áreas forestales de la Sierra Nevada de Texcoco, México.

\section{MATERIALES Y MÉTODOS}

\section{Área de estudio}

El área de estudio se ubica en la zona oriente del estado de México y corresponde a la zona montañosa ubicada en la porción oriental del municipio de Texcoco. Dicha área pertenece a la región fisiográfica conocida como Sierra Nevada, la cual forma parte del eje volcánico transversal (Sánchez y López, 2003). Aunque el área de interés es la zona de la Sierra Nevada bajo influencia del municipio de Texcoco, ésta se trabajó como parte de la zona de cobertura de la Carta "Chalco" del INEGI clave "E14B31" (INEGI, 1982), acotada por las coordenadas $19^{\circ} 15^{\prime} 00^{\prime \prime} \mathrm{N}$ a $19^{\circ} 30^{\prime} 00^{\prime \prime} \mathrm{N}$ y $98^{\circ} 40^{\prime} 00^{\prime \prime} \mathrm{O}$ a $99^{\circ} 00^{\prime} 00^{\prime \prime} \mathrm{O}$ (Fig. 1); la finalidad de procesar la información de la carta completa fue propiciar una mayor carga de operación al software para verificar su funcionamiento.

Carta E14B31, 1:50,000. "Chalco". INEGI.

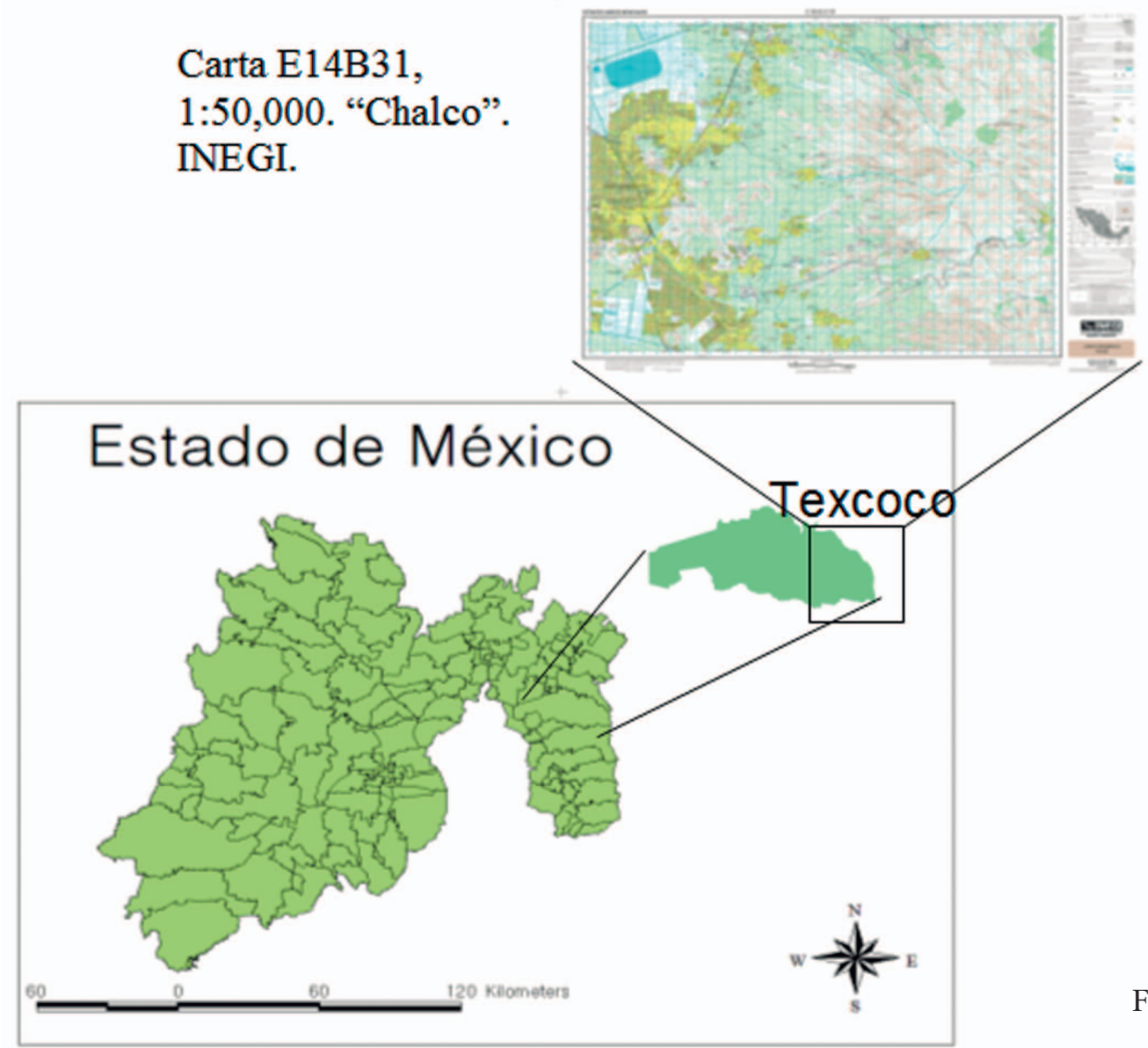

FiguRA 1. Área de estudio. 
En esta zona se presenta un intervalo altitudinal que va de los $2103 \mathrm{msnm}$ a los $4127 \mathrm{msnm}$ con una alta diversidad de paisajes cuyos principales tipos de vegetación son el bosque de pino, oyamel, pino-oyamel-encino, pino-aileencino, pino-encino y pastizal (Sánchez y López, 2003). La tenencia de los terrenos es uso común, bajo régimen ejidal, pertenecientes a las diferentes comunidades asentadas en las faldas de la sierra. Los terrenos han estado sujetos a aprovechamiento forestal bajo administración directa de los ejidos. Por la belleza de sus paisajes, la zona recibe gran afluencia de visitantes a lo largo del año, razón por la cual se ha pensado en el desarrollo de proyectos ecoturísticos como posible alternativa al aprovechamiento forestal (Martínez, 2005).

\section{Base de datos cartográfica}

Se utilizaron mapas digitales correspondientes a la carta Chalco "E14B31" de INEGI (1982) en escala 1:50 000 bajo un sistema de coordenadas UTM NAD27; los temas usados corresponden a los rasgos de áreas urbanas, vías de transportación, rasgos hidrográficos puntuales, corrientes y vías de conducción de agua y uso de suelo y vegetación, todos ellos en formato vectorial así como un modelo digital de elevaciones (MDE) con resolución espacial de $30 \mathrm{~m}$, en formato raster. En este sentido se hizo acopio y uso de la información existente; la generación de información temática de mayor detalle, aunque benéfica para su desarrollo, escapa a los objetivos del trabajo, recalcando que se trata de un estudio exploratorio.

\section{Proceso}

El proceso metodológico se describe en las cuatro etapas generales siguientes:

\section{Etapa 1. Conformación de la información de condiciones del ambiente}

Esta información se refiere a los requerimientos del ambiente necesarios para el desarrollo de una actividad ecoturística específica, expresados como una jerarquía de criterios y variables, así como a juicios de valor respecto de la importancia o prioridad relativa con la que cada varia- ble y criterio participa dentro del proceso de evaluación de aptitud. Las variables de interés fueron: distancia a núcleos de población y distancia a caminos bajo el criterio Accesibilidad; pendiente del terreno bajo el criterio Topografía; distancia a manantiales y distancia a cauces de agua bajo el criterio Agua; y tipo de vegetación bajo el criterio Vegetación. Éstas se obtuvieron mediante cuestionarios aplicados a personas que practican alguna actividad ecoturística.

\section{Diseño del cuestionario}

Para obtener la información de "condiciones del ambiente", se diseño, calibró y aplicó un cuestionario de 20 preguntas agrupadas en tres secciones: (a) Datos de identificación; (b) Información de condiciones del ambiente necesarias para practicar la actividad ecoturística de interés y (c) Valores de importancia o prioridad relativas para criterios y variables.

En la sección (a) del cuestionario, se propusieron actividades de campismo, caminata y día de campo; adicionalmente, se dejó opción para que el encuestado pudiera anotar cualquier otra actividad diferente a las anteriores y así poder indicar la actividad preferente para la cual aporta la información. En la sección (b) se incluyeron preguntas sobre condiciones del ambiente asociadas a rasgos cartografiables porque es requisito para el proceso que cada condición (variable) esté acompañada de la información temática correspondiente (en forma de mapas digitales). En la sección (c) se captó información de la participación de cada condición, ya sea criterio o variable, a través de un valor de importancia o prioridad.

Se aplicaron 46, 27 y 23 encuestas para las actividades ecoturísticas de campismo, caminata y día de campo, respectivamente; para ello no se definió un tamaño de muestra sino que se buscó al mayor número posible de personas que tuvieran cierto grado de conocimiento y experiencia de los requerimientos biofísicos y de facilidades para el desarrollo de las actividades ecoturísticas en la zona; de esta forma, la encuesta se aplicó a instructores de grupos de niños exploradores, profesores con estudios de licenciatura o postgrado en el área de turismo de las instituciones educativas del municipio, integrantes de una aso- 
ciación civil de caminata y estudiantes de último año en la carrera de turismo.

\section{Análisis de los cuestionarios}

El proceso de análisis se realizó por cada actividad ecoturística (campismo, caminata y día de campo). Para el análisis de las variables (sección b) se construyeron histogramas y se obtuvieron las estadísticas simples con la finalidad de explorar el comportamiento de los datos. Para cada variable se generaron intervalos con distintos niveles de recomendación (alta, media y baja) con base en las respuestas de los encuestados; dichos niveles representan una condición particular (aptitud) para el desarrollo de una actividad específica.

La generación de intervalos se basó en la función de distribución acumulativa empírica de cada variable en donde el término "empírica" hace referencia al hecho de que es generada con base en una muestra de la población
(Behar y Yepes, 2007). Los intervalos se definieron en términos de su probabilidad con la finalidad de asignarles un nivel de aptitud; así se establecieron intervalos de aptitud alta, media y baja asociados a probabilidades alta, media y baja respectivamente (Tabla 1). Para establecer los límites de los intervalos se usaron directamente los valores (registrados en los cuestionarios) con los que se generó la función de distribución.

La información de la sección (c) dio origen a los juicios de valor sobre la importancia o prioridad relativa asociados a cada criterio y variable. Para definir tales valores, se calculó el vector de prioridad y la razón de consistencia de la matriz de cada cuestionario, mediante la opción Weight del módulo Decision Support de IDRISI ${ }^{\oplus}$. Todas aquellas matrices cuya razón de consistencia fue mayor a 0,10 así como sus vectores, fueron descartados, debido a que tales valores indican una alta inconsistencia en los juicios de comparación en las matrices.

TABLA 1. Condiciones necesarias para la práctica de actividades ecoturísticas.

\begin{tabular}{|c|c|c|c|c|c|c|c|}
\hline \multirow[b]{2}{*}{ Condición } & \multirow[b]{2}{*}{ Aptitud } & \multicolumn{2}{|c|}{ Campismo } & \multicolumn{2}{|c|}{ Caminata } & \multicolumn{2}{|c|}{ Día de campo } \\
\hline & & Intervalo & $F$ & Intervalo & $F$ & Intervalo & $F$ \\
\hline \multirow{3}{*}{ Distancia a núcleos de población (m) } & $\mathrm{S} 1$ & $500-3000$ & 0,66 & $1000-1800$ & 0,63 & $100-1500$ & 0,69 \\
\hline & $\mathrm{S} 2$ & $3000-5000$ & 0,24 & $1800-5000$ & 0,31 & $1500-3000$ & 0,23 \\
\hline & S3 & $5000-10000$ & 0,1 & $5000-7500$ & 0,06 & $3000-5000$ & 0,08 \\
\hline \multirow{3}{*}{ Distancia a caminos (m) } & $\mathrm{S} 1$ & $10-500$ & 0,61 & $100-500$ & 0,59 & $75-500$ & 0,56 \\
\hline & $\mathrm{S2}$ & $500-1000$ & 0,26 & $500-1500$ & 0,35 & $500-1000$ & 0,38 \\
\hline & $\mathrm{s3}$ & $1000-4000$ & 0,13 & $1500-3000$ & 0,06 & $1000-2000$ & 0,06 \\
\hline \multirow{3}{*}{ Pendiente (\%) } & $\mathrm{S1}$ & $2.5-30$ & 0,61 & $0-45$ & 0,55 & $0-35$ & 0,64 \\
\hline & $\mathrm{s} 2$ & $30-75$ & 0,27 & $45-55$ & 0,35 & $35-60$ & 0,29 \\
\hline & S3 & $75-110$ & 0,12 & $55-90$ & 0,1 & $60-125$ & 0,07 \\
\hline \multirow{3}{*}{ Distancia a manantiales (m) } & $\mathrm{S1}$ & $5-350$ & 0,58 & $200-1000$ & 0,53 & $50-500$ & 0,72 \\
\hline & $\mathrm{s} 2$ & $350-1000$ & 0,3 & $1000-3500$ & 0,37 & $500-1500$ & 0,17 \\
\hline & S3 & $1000-5000$ & 0,12 & $3500-10000$ & 0,1 & $1500-2000$ & 0,11 \\
\hline \multirow{3}{*}{ Distancia a cauces (m) } & $\mathrm{S} 1$ & $5-200$ & 0,59 & $100-500$ & 0,58 & $20-400$ & 0,5 \\
\hline & $\mathrm{s} 2$ & $200-500$ & 0,27 & $500-2000$ & 0,26 & $400-500$ & 0,39 \\
\hline & 53 & $500-4000$ & 0,14 & $2000-4000$ & 0,16 & $500-1000$ & 0,11 \\
\hline \multirow{3}{*}{ Vegetación* } & $\mathrm{S} 1$ & Pino & 0,39 & Pino & 0,41 & Pino & 0,41 \\
\hline & $\mathrm{S2}$ & Encino & 0,35 & Oyamel & 0,31 & Encino & 0,32 \\
\hline & S3 & Oyamel & 0,26 & Encino & 0,28 & Cedro & 0,27 \\
\hline
\end{tabular}

S: Nivel de aptitud (SI=Alta; S2=Media; S3=Baja).

F: Probabilidad asociada al intervalo (De acuerdo con la Función de Distribución Acumulativa Empírica).

* En este caso se trata de una variable de tipo cualitativo referente a un tipo de vegetación. 
Posteriormente, los vectores de prioridad fueron incorporados en una gráfica conjunta de Excel $^{\circledR}$ de tipo líneas, en formato de series de datos para identificar visualmente los vectores extremos a la tendencia general; nuevamente, matrices y vectores extremos se descartaron para despreciar valores que se desvían marcadamente del comportamiento común de los datos y que darían origen a promedios no representativos.

Por último, de las matrices que fueron conservadas, se obtuvo un valor promedio para cada celda por encima de la diagonal de celdas con valor 1 y se volvió a verificar el índice de consistencia, constituyendo ésta, la matriz definitiva de valores de importancia o prioridad relativa para criterios a ser utilizada en el proceso de evaluación de aptitud (Tabla 2).

\section{Etapa 2. Conformación de la información temática}

Esta etapa consistió en realizar el procesamiento de la información temática previo al proceso de evaluación de aptitud a fin de proporcionarle las características necesarias para su ingreso al sistema. Dicho proceso consistió en lo siguiente:

- Creación de áreas de amortiguamiento de acuerdo con intervalos de aptitud (descritos en la sección anterior) para las capas temáticas de áreas urbanas, vías de transporte, rasgos hidrográficos puntuales, corrientes y vías de conducción de agua. Las vías de transporte en las áreas forestales son básicamente "brechas" y "veredas". Las brechas, al ser de mayor dimensión y permitir el paso de vehículos, pueden

TABLA 2. Matrices de comparación para estandarización de variables.*

\begin{tabular}{|c|c|c|c|c|c|c|c|c|c|c|c|c|}
\hline Condición & \multicolumn{4}{|c|}{ Campismo } & \multicolumn{4}{|c|}{ Caminata } & \multicolumn{4}{|c|}{ Día de campo } \\
\hline \multirow{4}{*}{ Distancia a núcleos de población } & & $\mathrm{S} 1$ & $\mathrm{~s} 2$ & S3 & & $\mathrm{S} 1$ & S2 & S3 & & $\mathrm{S} 1$ & $\mathrm{~s} 2$ & S3 \\
\hline & $\mathrm{S} 1$ & 1 & 2,75 & 6,6 & S1 & 1 & 2,03 & 10,5 & $\mathrm{S1}$ & 1 & 3 & 8,63 \\
\hline & $\mathrm{S} 2$ & & 1 & 2,4 & $\mathrm{~S} 2$ & & 1 & 5,17 & $\mathrm{~S} 2$ & & 1 & 2,88 \\
\hline & S3 & & & 1 & S3 & & & 1 & S3 & & & 1 \\
\hline \multirow{4}{*}{ Distancia a caminos } & & $\mathrm{S} 1$ & $\mathrm{~S} 2$ & S3 & & $\mathrm{S} 1$ & $\mathrm{~S} 2$ & S3 & & $\mathrm{S1}$ & $\mathrm{S} 2$ & S3 \\
\hline & $\mathrm{S1}$ & 1 & 2,35 & 4,69 & $\mathrm{~S} 1$ & 1 & 1,69 & 9,83 & $\mathrm{~S} 1$ & 1 & 1,47 & 9,33 \\
\hline & $\mathrm{s} 2$ & & 1 & 2 & $\mathrm{~s} 2$ & & 1 & 5,83 & $\mathrm{~S} 2$ & & 1 & 6,33 \\
\hline & 53 & & & 1 & S3 & & & 1 & S3 & & & 1 \\
\hline \multirow{4}{*}{ Pendiente } & & $\mathrm{S} 1$ & $\mathrm{S2}$ & $\mathrm{S3}$ & & $\mathrm{S} 1$ & S2 & S3 & & $\mathrm{S} 1$ & $\mathrm{~s} 2$ & S3 \\
\hline & $\mathrm{Sl}$ & 1 & 2,26 & 5,08 & $\mathrm{~S} 1$ & 1 & 1,57 & 5,5 & $\mathrm{~S} 1$ & 1 & 2,21 & 9,14 \\
\hline & 52 & & 1 & 2,25 & $\mathrm{~s} 2$ & & 1 & 3,5 & $\mathrm{S2}$ & & 1 & 4,14 \\
\hline & 53 & & & 1 & S3 & & & 1 & S3 & & & 1 \\
\hline \multirow{4}{*}{ Distancia a manantiales } & & $\mathrm{S} 1$ & $\mathrm{~s} 2$ & $\mathrm{s3}$ & & $\mathrm{S1}$ & $\mathrm{S} 2$ & S3 & & $\mathrm{S} 1$ & $\mathrm{~s} 2$ & S3 \\
\hline & $\mathrm{S1}$ & 1 & 1,93 & 4,83 & $\mathrm{~S} 1$ & 1 & 1,43 & 5,3 & $\mathrm{S1}$ & 1 & 4,24 & 6,55 \\
\hline & $\mathrm{S} 2$ & & 1 & 2,5 & $\mathrm{~S} 2$ & & 1 & 3,7 & $\mathrm{~S} 2$ & & 1 & 1,55 \\
\hline & 53 & & & 1 & S3 & & & 1 & S3 & & & 1 \\
\hline \multirow{4}{*}{ Distancia a cauces } & & $\mathrm{S} 1$ & $\mathrm{~S} 2$ & S3 & & $\mathrm{S1}$ & $\mathrm{s} 2$ & S3 & & $\mathrm{S1}$ & $\mathrm{S} 2$ & $\mathrm{S3}$ \\
\hline & $\mathrm{Sl}$ & 1 & 2,19 & 4,21 & S1 & 1 & 2,23 & 3,63 & $\mathrm{~S} 1$ & 1 & 1,28 & 4,55 \\
\hline & $\mathrm{S2}$ & & 1 & 1,93 & $\mathrm{S2}$ & & 1 & 1,63 & $\mathrm{~S} 2$ & & 1 & 3,55 \\
\hline & S3 & & & 1 & S3 & & & 1 & S3 & & & 1 \\
\hline \multirow{4}{*}{ Vegetación } & & $\mathrm{S1}$ & $\mathrm{S} 2$ & S3 & & $\mathrm{S} 1$ & $\mathrm{~S} 2$ & S3 & & $\mathrm{S} 1$ & $\mathrm{~S} 2$ & S3 \\
\hline & $\mathrm{S1}$ & 1 & 1,11 & 1,5 & $\mathrm{~S} 1$ & 1 & 1,29 & 1,47 & $\mathrm{~S} 1$ & 1 & 1,31 & 1,55 \\
\hline & $\mathrm{S} 2$ & & 1 & 1,34 & $\mathrm{~S} 2$ & & 1 & 1,13 & $\mathrm{~S} 2$ & & 1 & 1,18 \\
\hline & S3 & & & 1 & S3 & & & 1 & S3 & & & 1 \\
\hline
\end{tabular}

* Las celdas vacías por debajo de la diagonal de valores 1 aparecen sin valor debido a que su valor está determinado por el valor inverso de su celda de comparación recíproca. 
generar un flujo constante de tránsito, lo que podría representar un inconveniente (al generar ruido, polvo o simplemente perturbar la calma) para la realización de actividades ecoturísticas. Por tal razón, éste fue el tipo de vía utilizada para crear las áreas de amortiguamiento. Para el caso de rasgos hidrográficos puntuales, las áreas de amortiguamiento se crearon sólo con respecto a los manantiales y para el caso de corrientes y vías de conducción de agua, sólo sobre corrientes permanentes.

- Recorte y conversión de los mapas de áreas de amortiguamiento de formato vectorial a raster tomando como base las dimensiones del mapa de pendientes.

- Generación del mapa de pendientes a partir del MDE y reclasificación de acuerdo con intervalos de aptitud.

- Reproyección del mapa de pendientes de datum WGS84 a NAD27.

- Conversión de mapas raster en formato grid de ArcView a formato de archivo ASCII.

\section{Etapa 3. Procesamiento}

Esta etapa consistió en la puesta en operación del software (Pérez et al., 2013) alimentándolo con la información generada tanto en la primera como en la segunda etapa y siguiendo para ello las instrucciones del manual de usuario (Pérez et al., 2011). El sistema realiza tres procesos generales: (a) El primero corresponde a la conformación del Proceso Jerárquico Analítico (PJA) de Evaluación Multicriterio, mediante el cual se generan valores para estandarizar variables, ponderar criterios y verificar la consistencia de matrices de comparación (Malczewski, 1999); (b) El segundo corresponde a la modelación cartográfica, que comprende utilizar los valores generados en el proceso PJA (paso anterior) para generar los mapas temáticos estandarizados y los mapas ponderados tanto de variables como de criterios, y después combinar los mapas en cada nivel de la jerarquía para sintetizar el mapa, desde el nivel inferior (variables) hasta el superior (aptitud); y (c) El tercer proceso retoma el mapa de aptitud y nuevamente, mediante modelación cartográfica reclasifica el mapa de aptitud en clases establecidas a juicio del usuario, opcionalmente el mapa de aptitud puede ser combinado con un mapa binario de restricciones de uso de suelo para descartar las áreas impedidas para el desarrollo de la actividad ecoturística.

\section{Etapa 4. Prueba del sistema}

Una tarea relevante en el desarrollo de software es asegurar que éste satisfaga las necesidades del usuario. Un elemento de gran importancia dentro del proceso de control de desarrollo de software es su validación o prueba; para su aplicación se requiere disponer de un prototipo ejecutable el cual se pone en marcha con la finalidad de contrastar sus respuestas con el comportamiento operacional esperado, en donde los resultados son examinados para constatar su fiabilidad y así probar que el sistema es de utilidad operacional. Para realizar una prueba, es necesario definir las pre-condiciones y post-condiciones, establecer valores de entrada y conocer el comportamiento que debería tener el sistema ante dichos valores (INTECO, 2009).

En ese sentido, la prueba del sistema se realizó en dos fases: (i) Comprobación de la lógica de operación del sistema. Para esta operación se generaron seis capas de datos en formato raster, con dimensión de 9 filas por 9 columnas. Estas capas se utilizaron para realizar diferentes ejecuciones del sistema. En cada ejecución se asignaron valores diversos (bien conocidos) a una de las capas, manteniendo las restantes en un valor fijo (el de mayor aptitud en todas sus celdas) para poder conocer con certeza el valor que debía ser esperado y contrastarlo con el generado por el sistema, y así poder comprobar su correcto desempeño; (ii) La segunda etapa consistió en la verificación manual del valor de aptitud para una serie de puntos del área de estudio para cada actividad y su comparación con el valor generado por el sistema, a fin de verificar su confiabilidad. Los puntos fueron definidos mediante la generación de valores aleatorios para sus coordenadas $\mathrm{X}$ y Y aplicando la función "aleatorio.entre()" de Excel$^{\circledR}$; se consideró un total de 15 puntos por actividad.

\section{RESULTADOS Y DISCUSIÓN}

A través de las encuestas se definieron las condiciones necesarias (en forma de intervalos de aptitud) en cuanto a 
distancia, núcleos de población, distancia a caminos, pendiente del terreno, distancia a manantiales y distancia a cauces de agua necesarias para la práctica de las actividades campismo, caminata y día de campo en la Sierra Nevada de Texcoco (Tabla 1). Cabe mencionar que las personas a las que se aplicó la encuesta representaron solo un pequeño grupo de muestra de la inmensa mayoría que componen a la sociedad; este estudio en su naturaleza exploratoria generó, de esta muestra, la información necesaria para aplicar la metodología. Pero es claro que se requieren de estudios complementarios que permitan profundizar en el universo de gustos y preferencias de los diferentes grupos sociales, en donde se conjugan aspectos como el género, edad, situación económica, nivel cultural, etc. Una situación así permitiría el planteamiento de escenarios bien definidos en cuanto a ponderaciones de criterios y variables con respecto a cada grupo, prescindiendo de esta manera del análisis de sensibilidad que suele acompañar a las herramientas de evaluación multicriterio.

Una consideración importante en cuanto a las actividades ecoturísticas es que, a pesar de que el cuestionario incluía opción para mencionar actividades diferentes a las que se proponían en el mismo, ningún cuestionario registró actividades adicionales. Esto no quiere decir que no existan otras actividades susceptibles de desarrollarse en la zona sino que para la muestra encuestada, estas fueron las actividades más comunes. En su carácter exploratorio, no estuvo en los alcances de este trabajo realizar un análisis exhaustivo de todas y cada una de las actividades que pueden desarrollarse en el área de estudio; desde luego este tema de gran relevancia tendrá que abordarse a través de estudios complementarios.

El nivel de aptitud asociado con los intervalos indica una situación de idealidad para la práctica de las actividades ecoturísticas. Para este trabajo en particular se establecieron los niveles de aptitud nula, baja, media y alta, pero puede establecerse cualquier número de niveles, lo que dependerá de los objetivos particulares de la investigación. En este sentido, el sistema de evaluación permite definir cualquier número de niveles.

Con base en los intervalos de aptitud para cada condición (variable) se establecieron las matrices de compara-

TABLA 3. Matrices de comparación para ponderación de subcriterios (variables).*

\begin{tabular}{|c|c|c|c|c|c|c|c|}
\hline Criterio & \multirow[b]{2}{*}{ Variable } & \multicolumn{2}{|c|}{ Campismo } & \multicolumn{2}{|c|}{ Caminata } & \multicolumn{2}{|c|}{ Día de campo } \\
\hline \multirow{3}{*}{ Accesibilidad } & & $\begin{array}{c}\text { Distancia a } \\
\text { caminos }\end{array}$ & $\begin{array}{c}\text { Distancia a } \\
\text { núcleos de } \\
\text { población }\end{array}$ & $\begin{array}{c}\text { Distancia a } \\
\text { caminos }\end{array}$ & $\begin{array}{c}\text { Distancia } \\
\text { a núcleos } \\
\text { de pobla- } \\
\text { ción }\end{array}$ & $\begin{array}{l}\text { Distancia } \\
\text { a caminos }\end{array}$ & $\begin{array}{c}\text { Distancia a } \\
\text { núcleos de } \\
\text { población }\end{array}$ \\
\hline & Distancia a caminos & 1 & 0,21 & 1 & 3,66 & 1 & 0,25 \\
\hline & $\begin{array}{l}\text { Distancia a núcleos de } \\
\text { población }\end{array}$ & & 1 & & 1 & & 1 \\
\hline \multirow{3}{*}{ Agua } & Variable & $\begin{array}{l}\text { Distancia a } \\
\text { cauces }\end{array}$ & $\begin{array}{l}\text { Distancia a } \\
\text { manantiales }\end{array}$ & $\begin{array}{l}\text { Distancia a } \\
\text { cauces }\end{array}$ & $\begin{array}{l}\text { Distancia } \\
\text { a manan- } \\
\text { tiales }\end{array}$ & $\begin{array}{l}\text { Distancia } \\
\text { a cauces }\end{array}$ & $\begin{array}{l}\text { Distancia a } \\
\text { manantiales }\end{array}$ \\
\hline & Distancia a cauces & 1 & 0,429 & 1 & 3 & 1 & 0,142 \\
\hline & Distancia a manantiales & & 1 & & 1 & & 1 \\
\hline
\end{tabular}

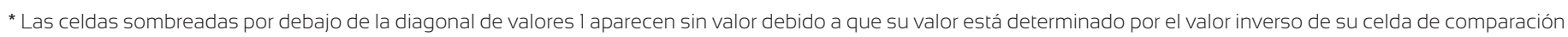
recíproca. 
ción para su estandarización (Tabla 2). Los valores de probabilidad, ya en sí, representan valores de estandarización, pero para acoplarlos al formato de entrada del sistema, que son las matrices de comparación, se obtuvo el cociente de los valores de probabilidad de los niveles que se comparan; dicho cociente representa la dimensión proporcional de uno frente al otro. Cuando se resuelve la matriz de comparación generando el vector de prioridades, éste retorna los valores de probabilidad originales; un paso posterior es la obtención del cociente de cada valor del vector con respecto al mayor de ellos para dar origen a los valores de estandarización. También se conformaron las matrices de comparación para ponderación de variables al interior de criterios -subcriterios- (Tabla 3) así como para ponderación de criterios (Tabla 4). Es necesario comentar que en la tabla 3 no figuran los criterios “Topografía” y "Vegetación” porque no se están ponderando dado que no tienen más de una variable.

El sistema fue puesto en ejecución con base en el manual de operación (Pérez et al., 2011), incorporando las capas temáticas necesarias, generando la jerarquía de criterios (Fig. 2a), incorporando los intervalos de aptitud con sus valores de importancia (Fig. 2b), así como los valores de importancia de variables (Fig. 2c) y de criterios (Fig. 2d); el proceso fue desarrollado para cada actividad ecoturística para obtener el mapa de aptitud correspondiente.

De acuerdo con los criterios y subcriterios, así como con el formato de la información empleada en el proceso, el sistema puede generar para estas condiciones alrededor de 4096 posibles valores de aptitud. Por tal razón, se realizó la reclasificación de los mapas de aptitud para las tres actividades de interés en las clases de Aptitud: Nula= $(0 \leq$ Aptitud $\leq 0,5), \quad$ Baja $=(0,5<$ Aptitud $\leq 0,66), \quad$ Media $=$ $(0,66<$ Aptitud $\leq 0,83)$ y Alta $=(0,83<$ Aptitud $\leq 1,0)$.

De esta manera, el sistema generó los mapas de aptitud para las actividades de campismo, caminata y día de campo (Figs. 3, 4 y 5, respectivamente) donde se aprecian las clases de aptitud mencionadas y se presentan adicionalmente zonas de restricción -en este caso corresponden a áreas urbanas-; la inclusión de zonas de restricción es otra funcionalidad del sistema. La inclusión de áreas urbanas como zonas de restricción solo es un parámetro de referencia debido a que no se cuenta con información actualizada.

Además, se percibe que tales zonas han ido en constante aumento; por la misma razón no se incluyeron otras zonas de restricción como las de uso agrícola. Queda confirmado que el sistema permite la incorporación de cual-

TABLA 4. Matriz de comparación para ponderación de criterios.*

\begin{tabular}{|c|c|c|c|c|c|}
\hline & & Accesibilidad & Agua & Topografía & Vegetación \\
\hline \multirow{4}{*}{ Campismo } & Accesibilidad & & 0,22 & 2,58 & 1,17 \\
\hline & Agua & & 1 & 4,34 & 4,5 \\
\hline & Topografía & & & 1 & 1,17 \\
\hline & Vegetación & & & & 1 \\
\hline \multirow{4}{*}{ Caminata } & Accesibilidad & & 2 & 3,67 & 4,33 \\
\hline & Agua & & 1 & 2,33 & 5 \\
\hline & Topografía & & & 1 & 4,33 \\
\hline & Vegetación & & & & 1 \\
\hline \multirow{4}{*}{ Día de campo } & Accesibilidad & & 3 & 0,51 & 1,67 \\
\hline & Agua & & 1 & 0,29 & 0,56 \\
\hline & Topografía & & & 1 & 3 \\
\hline & Vegetación & & & & 1 \\
\hline
\end{tabular}

* Las celdas sombreadas por debajo de la diagonal de valores 1 aparecen sin valor debido a que su valor está determinado por el valor inverso de su celda de comparación recíproca. 


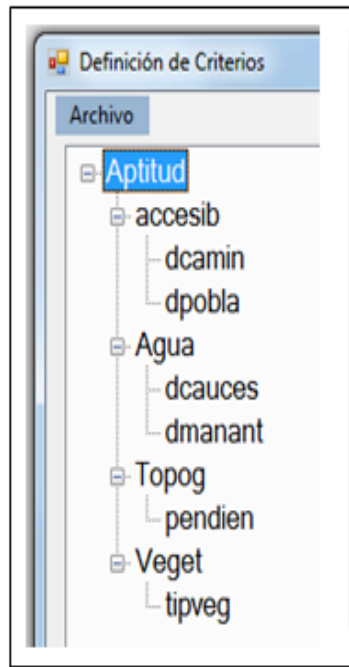

(a)

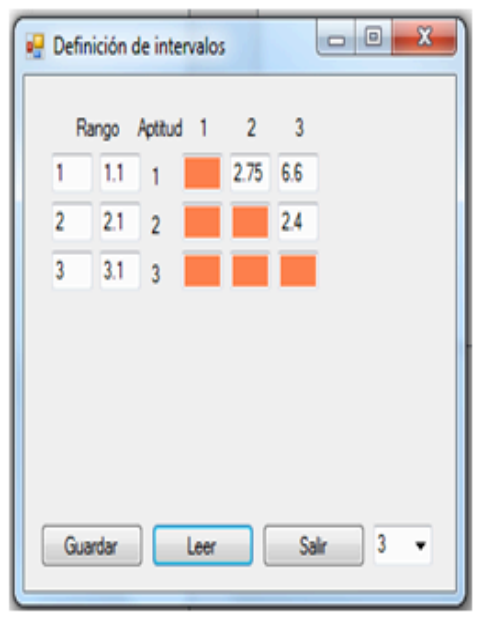

(b)

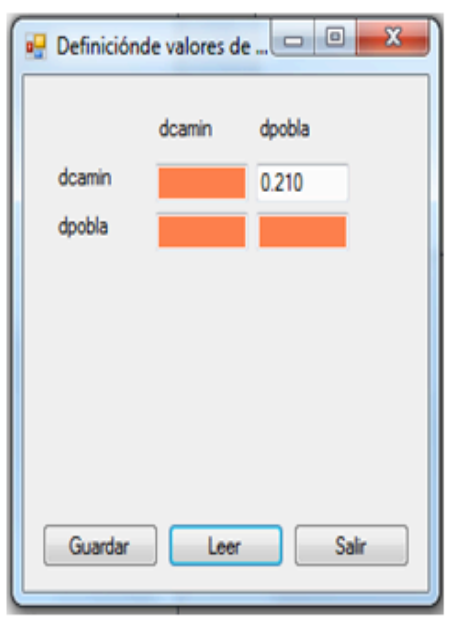

(c)

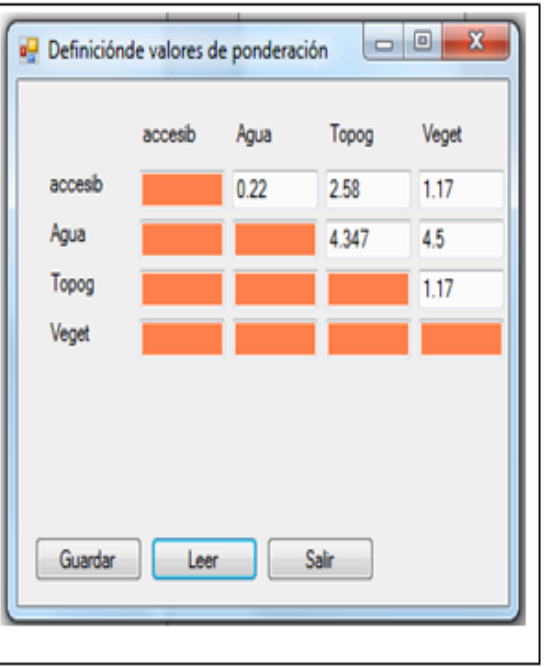

(d)

FIGURA 2. Ventanas del sistema para la incorporación de jerarquía de criterios (a), intervalos de aptitud (b), valores de importancia de variables (c), y valores de importancia de criterios (d).

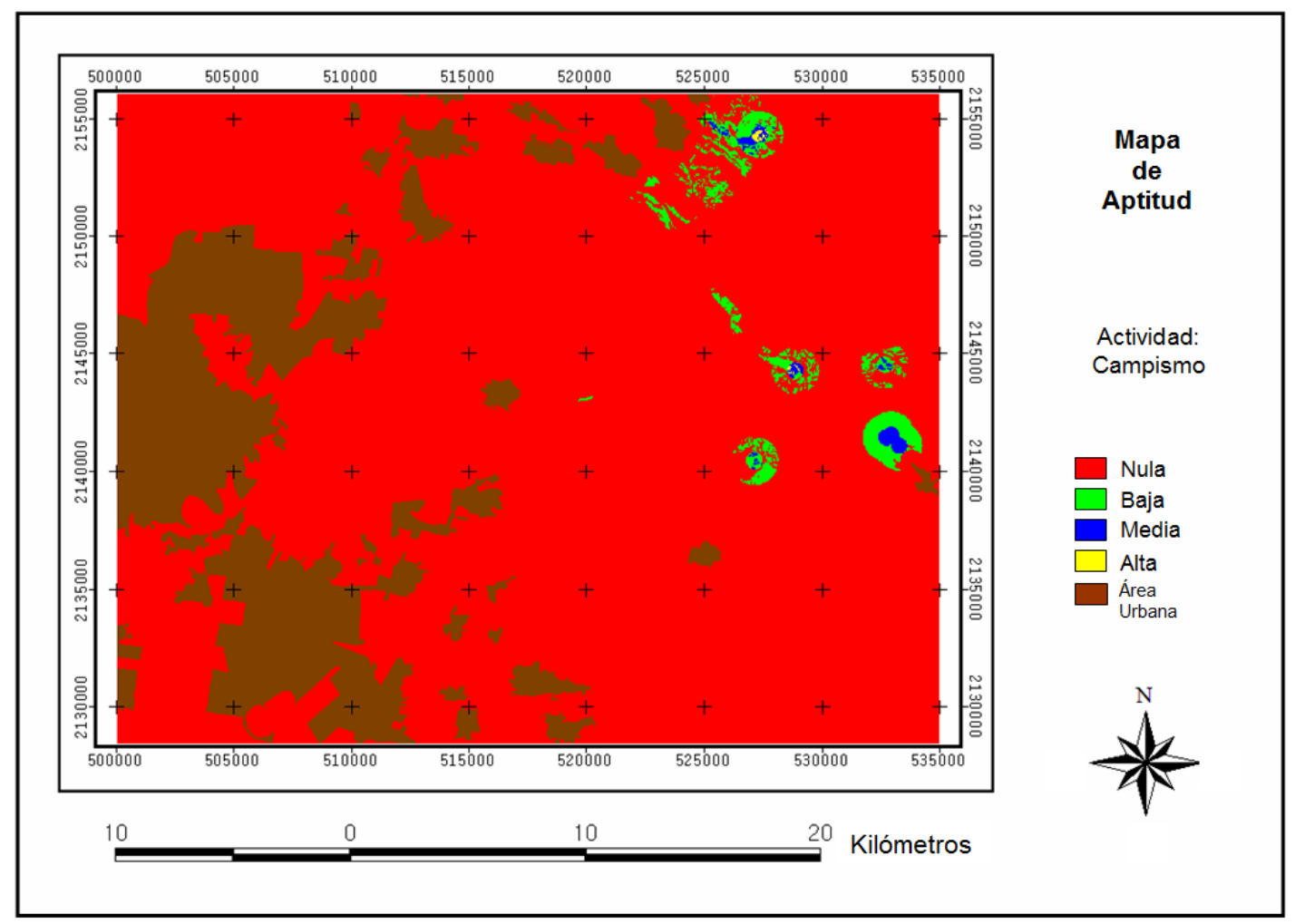

FIGURA 3. Mapa de aptitud para actividad de campismo. 


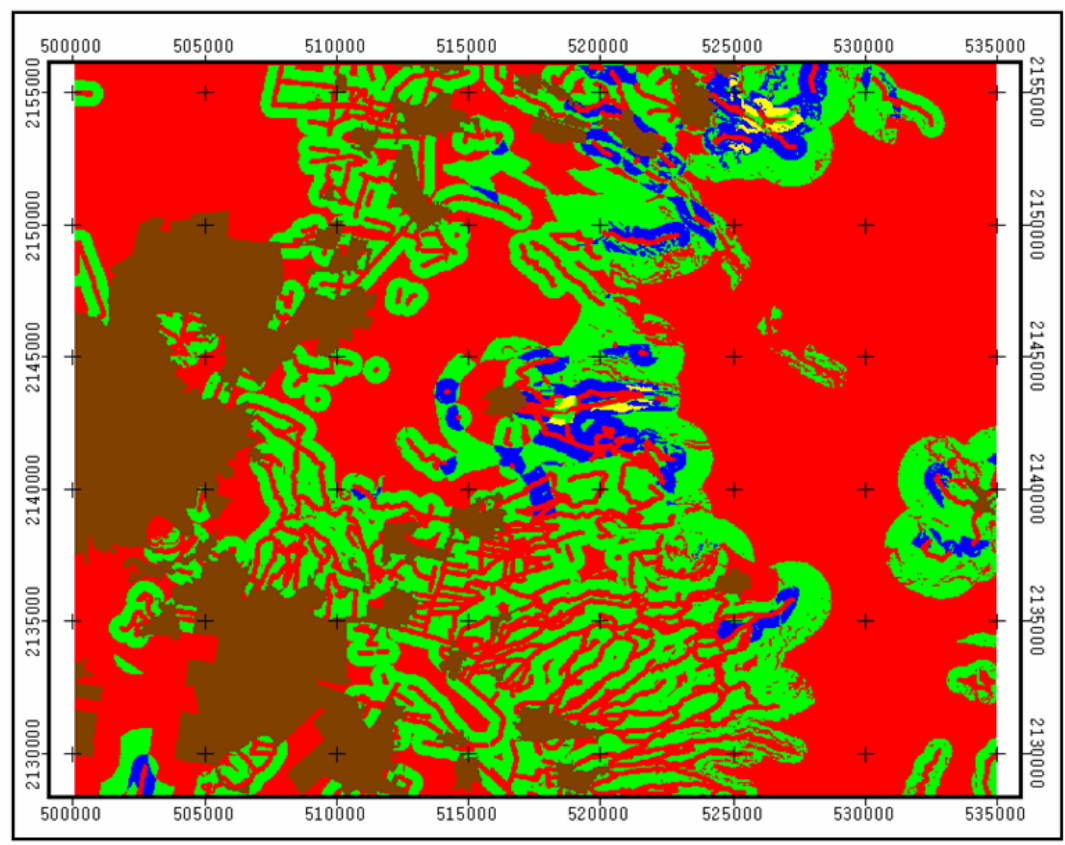

20 Kilómetros

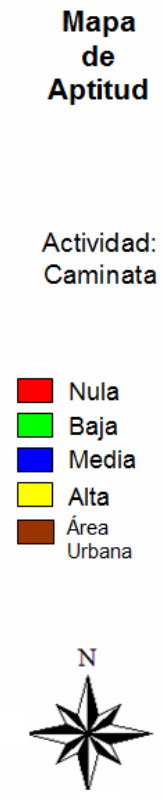

FIGURA 4. Mapa de aptitud para actividad de caminata.

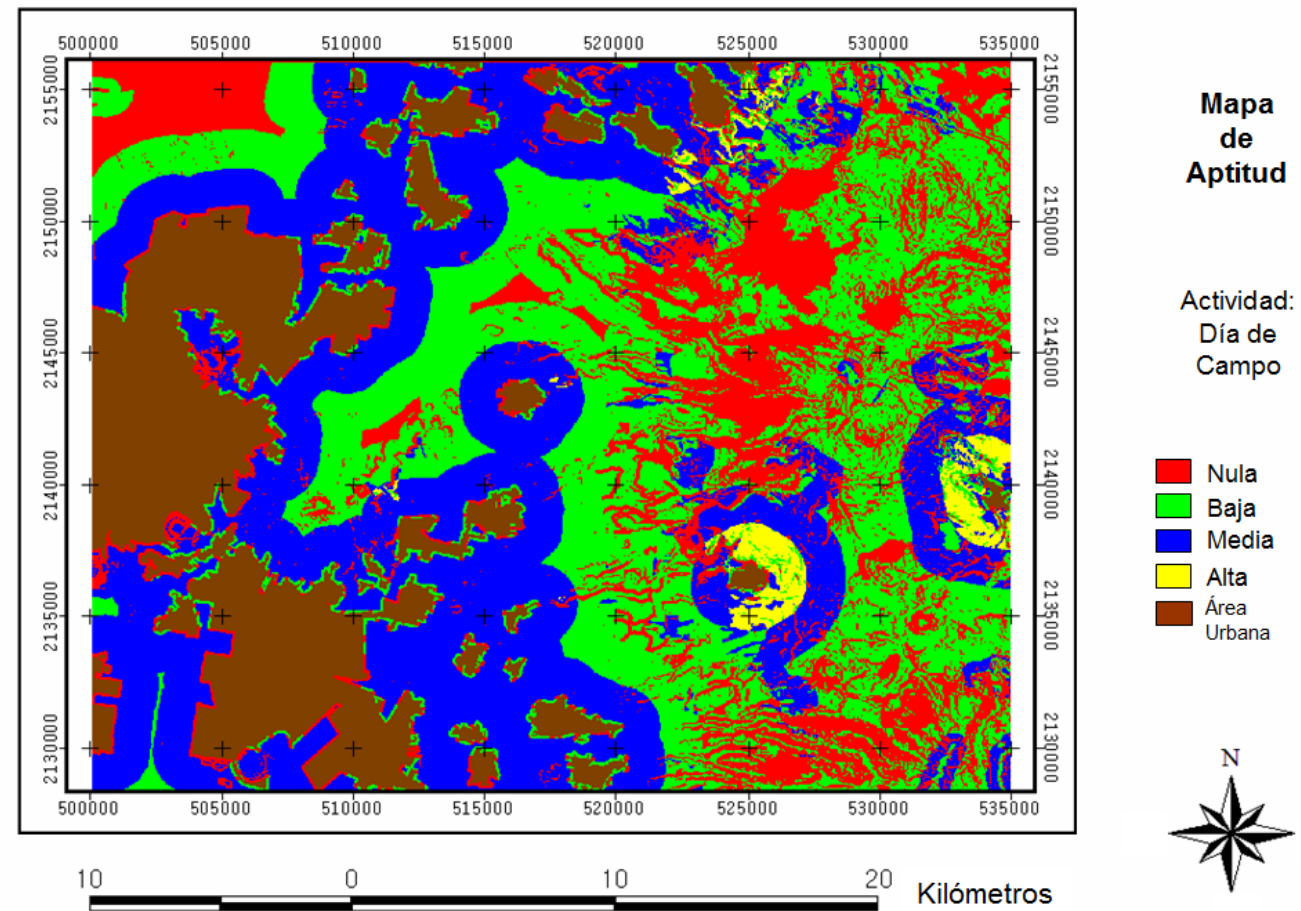

FIGURA 5. Mapa de aptitud para actividad de día de campo. 
quier número de zonas de restricción. Un aspecto de gran relevancia es la disponibilidad de información temática y su calidad. Como puede apreciarse, la utilidad de una evaluación como la que se realiza en el presente trabajo estará limitada por estos dos componentes. Si bien es cierto que este trabajo presenta tal limitación, su verdadero mérito lo constituye el presentar un enfoque de evaluación de aptitud (diferente al de trabajos relacionados al tema) que en consecuencia conlleva una metodología particular y adicionalmente presenta un sistema en este tipo de evaluaciones.

Los mapas indicaron una superficie con aptitud alta de 24,$3 ; 256,68$ y 1396,17 hectáreas para las actividades de campismo, caminata y día de campo, respectivamente (Tabla 5). Como se observa, la superficie para día de campo supera con mucho a las otras actividades, así la superficie para campismo y caminata representan $1,74 \%$ y $18,38 \%$ con respecto a la superficie de día de campo respectivamente. Las cantidades de superficie así como su distribución son consecuencia directa de las condiciones definidas para las actividades; así puede intuirse que las condiciones para campismo fueron muy restrictivas quedando en segundo término las correspondientes a caminata. De esto se deriva que la actividad de campismo es una actividad de mayor especialización que requiere de condiciones muy particulares y que, en sentido contrario, la actividad de día de campo está abierta a un margen bastante amplio de condiciones, observándose así la mayor vocación de la zona para actividades de día de campo.
Respecto a la prueba del sistema, tanto la comprobación de la lógica de operación como la verificación manual de valores de puntos aleatorios resultaron satisfactorias en toda su extensión, al no encontrar ningún valor diferente a los esperados; por tanto, la confiabilidad del sistema queda constatada.

El valor de aptitud tiene un significado fácilmente comprensible, el valor 1 indica una condición de idealidad, la cual disminuye en la medida en que se aproxima a 0 ; sin embargo, resume una enorme cantidad de información acerca de las relaciones complejas existentes entre los elementos que se conjugan para su determinación. Por esa razón, en la medida en que tales relaciones puedan ser acertadamente definidas en la información de entrada, el valor de aptitud se acercará a la realidad.

En este sentido, el sistema posee gran potencial para la representación de relaciones complejas; sin embargo, tal potencial se verá limitado en función de la cantidad y calidad de la información cartográfica disponible. Otra ventaja del sistema es que puede operar a cualquier nivel de escala, restringida por el aspecto de la información mencionada.

La información requerida por el sistema (Tabla 6) es un aspecto crítico debido a que podría representar una seria desventaja para el mismo; en este sentido, la confiabilidad del sistema dependerá completamente del grado en que la información cartográfica coincida con la realidad; un segundo punto es que si no hay disponibilidad de la

TABLA 5. Cuantificación de superficie por nivel de aptitud para cada actividad ecoturística.

\begin{tabular}{lrrrrrr}
\hline & \multicolumn{2}{c}{ Acampar } & \multicolumn{2}{c}{ Caminata } & \multicolumn{2}{c}{ Día de campo } \\
\hline \multicolumn{1}{c}{ Aptitud } & Superficie (ha) & \multicolumn{1}{c}{$\%$} & Superficie (ha) & \multicolumn{1}{c}{$\%$} & Superficie (ha) & \multicolumn{1}{c}{$\%$} \\
\hline Nula & 78679,08 & 81,55 & 50522,04 & 52,36 & 20328,84 & 21,07 \\
\hline Baja & 1326,15 & 1,37 & 26270,37 & 27,23 & 28786,23 & 29,84 \\
\hline Media & 211,41 & 0,22 & 3191,85 & 3,31 & 29729,70 & 30,81 \\
\hline Alta & 24,30 & 0,03 & 256,68 & 0,27 & 1396,17 & 1,45 \\
\hline Zona Urbana & 16243,02 & 16,83 & 16243,02 & 16,83 & 16243,02 & 16,83 \\
\hline Superficie total & 96483,96 & $100 \%$ & 96483,96 & $100 \%$ & 96483,96 & $100 \%$ \\
\hline
\end{tabular}


información correspondiente a la condiciones que se evalúan, la representación de la situación que se pretende evaluar quedará muy limitada. Ambos aspectos marcarán la utilidad del sistema.

TABLA 6. Resumen de la información requerida por el software para el análisis de aptitud.

\section{Variables ambientales}

\begin{tabular}{l}
$\begin{array}{l}\text { Valores de ponderación e intervalos de aptitud de variables } \\
\text { ambientales }\end{array}$ \\
\hline Definición de criterios \\
\hline Valores de ponderación para criterios \\
\hline Definición de Estructura jerárquica de criterios y variables \\
\hline Información temática correspondiente a variables ambien- \\
tales \\
Mapas de restricción de uso del suelo
\end{tabular}

\section{CONCLUSIONES}

La combinación de herramientas multicriterio y sistemas de información geográfica integradas en un sistema de cómputo representan una alternativa objetiva y confiable para la determinación de aptitud de áreas forestales para la realización de actividades ecoturísticas.

Mediante el sistema de cómputo se determinó que La Sierra Nevada de Texcoco cuenta con áreas naturales susceptibles de ser aprovechadas para realizar actividades ecoturísticas como campismo, caminata y día de campo; se observó una mayor aptitud para la actividad de Día de Campo, ya que entre las categorías "Media" y "Alta", más de $30 \%$ del área total de la zona de estudio presenta aptitud para esta actividad.

El sistema de cómputo diseñado permitió identificar zonas de aptitud ecoturística con base en criterios establecidos, y puede tener aplicación como herramienta auxiliar en otras áreas de interés, como en el ordenamiento ecológico para la búsqueda de patrones de ocupación del territorio y orientación de las políticas de uso del suelo; en el área de servicios ecosistémicos para identificar zonas para captura de carbono, captación de agua, protección de biodiversidad, etc.; en protección civil para identificar zonas de riesgos o para emplazamiento de infraestructura; entre otras, representando así una valiosa herramienta de apoyo a los procesos de toma de decisiones en la planificación ambiental.

Finalmente es importante recalcar, en los términos que lo plantea Senol et al. (2003), que el uso de los espacios naturales debe estar sustentado en bases objetivas como la cuantificación del potencial de sus atributos bióticos y abióticos para sustentar algún tipo de actividad, como una condición indispensable para encaminarse al uso racional y sustentable de los recursos naturales.

\section{REFERENCIAS}

Behar, R. y M. Yepes. 2007. Estadística. Un enfoque descriptivo. Universidad del Valle. ISBN 958-670-068-2. Cali, Colombia. $140 \mathrm{p}$.

Bandyopadhyay, S., R.K. Jaiswal, V.S. Hegde y V. Jayaraman. 2009. Assessment of land suitability potentials for agriculture using a remote sensing and GIS based approach. International Journal of Remote Sensing 30(4):879-895.

Blancas P., F.J., F.M. Guerrero C. y M. Lozano O. (2009). La localización espacial en la planificación del turismo rural en Andalucía: Un enfoque multicriterio. Revista de Estudios Regionales 48:83-113.

Bojórquez T., L.A., S. Díaz M. y P. Gómez P. 1999. GIS-approach for land sutability assessment in developing countries: A case study of forest development project in Mexico. In: H. Jean C., ed. Spatial multicriteria decision making and analisys: A geographic information sciences approach. Ashgate Publishing Company. p:335-353.

Caballero R., A.E. 2007. Los programas y proyectos turísticos del municipio de Texcoco, una alternativa viable para el desarrollo. Tesis licenciatura. Universidad Autónoma Chapingo. 86 p.

Ceballos L., H. 1998. Ecoturismo. Naturaleza y desarrollo sostenible. Diana. México. 185 p.

Chávez De la P., J. 2005. Ecoturismo TAP. Turismo ambientalmente planificado. Trillas. México. 138 p.

De Groot, R. 2006. Function-analisys and valuation as a tool assess land uses conflicts in planning for sustainable, multi-functional landscapes. Landscape and urban planning 75(3-4): 75-186. 
Franco M., S., M. Osorio G., G. Nava B. y H.H. Regil G. 2009. Evaluación multicriterio de los recursos turísticos. Parque Nacional Nevado de Toluca, México. Estudios y Perspectivas en Turismo 18:208-226.

Gómez B., E. y R. De Groot. 2007. Capital natural y funciones de los ecosistemas: Explorando las bases ecológicas de la economía. Ecosistemas XVI(3):4-14.

INEGI (Instituto Nacional de Estadística y Geografí). 1982. Carta Topográfica E14B31 (Chalco) Escala 1:50 000. Instituto Nacional de Estadística, Geografía e Informática. México, D.F.

INTECO (Instituto Nacional de Tecnologías de la Comunicación). 2009. Guía de validación y verificación. Laboratorio Nacional de Calidad del Software. Instituto Nacional de Tecnologías de la Comunicación. Gobierno de España. $81 \mathrm{p}$.

Kimmel, J.R. 1999. Ecotourism as environmental learning. The Journal of Environmental Education 30(2):40-44.

Kenan, O. 2006. Multiple criteria activity selection for ecotourism planning in Igneada. Turkish Journal of Agriculture and Forestry 30:153-164.

López H., N. y A. Triviño P. 2004. Planificación y gestión sostenible de áreas recreativas en la comunidad Valenciana. Cuadernos Geográficos 34:163-178.

Malczewski, J. 1999. GIS and multicriteria decision analysis. John Wiley \& Sons. EUA. 392 p.

Martínez R., M.L. 2005. La identidad cultural y el sistema de cargos como ejes de un proyecto intercomunitario en las comunidades de la Sierra Nevada de Texcoco, México. Tesis de doctorado. Universidad Autónoma Chapingo. $310 \mathrm{p}$.

Pérez de las Heras, M. 2003. La guía del ecoturismo: o cómo conservar la naturaleza a través del turismo. Edit. MundiPrensa. Madrid, España. 290 p.

Pérez V., M.A.; M.J. González G., J.R. Valdez L., H.M. De Los Santos P., G. Ángeles P. 2011. Manual de usuario: Sistema de información para determinar la aptitud ecoturística de áreas forestales. Colegio de Postgraduados. Montecillo, México. 28 p.

Pérez V., M.A.; M.J. González G., J.R. Valdez L. 2012. Métodos para determinar la aptitud ecoturística de áreas forestales. Revista Chapingo. Serie Ciencias Forestales y del Ambiente 18(3):271-189.

Pérez V., M.A.; M.J. González G., J.R. Valdez L., H.M. De Los Santos P., G. Ángeles P. 2013. Diseño de un sistema de cómputo para determinar la aptitud ecoturística de áreas forestales. Revista Chapingo. Serie Ciencias Forestales y del Ambiente 19(1):13-28.

Romero, C. 1993. Teoría de la decisión multicriterio: Conceptos, técnicas y aplicaciones. Alianza Editorial. Madrid, España. 191 p.

Sánchez G., A. y L. López M. 2003. Clasificación y ordenación de la vegetación del norte de la Sierra Nevada a lo largo de un gradiente altitudinal. Anales del Instituto de Biología, Serie Botánica 74(1):47-71.

Saaty, T.L. 1990. How to make a decision. The analytic hierarchy process. European Journal of Operational Research $48(1): 9-26$

Senol, S., S. Kilic y F. Evrendilek. 2003. Evaluation of land use potential and suitability of ecosystems in Antakya for reforestation, recreation, arable farming and residence. Turkish Journal of Agriculture and Forestry 27(1):15-22.

Wunder, S. 1999. Promoting forest conservation through ecotourism income? A case study from the Ecuadorian Amazon region. CIFOR. Ocasional paper No. 21. 24 p.

Manuscrito recibido el 20 de noviembre de 2012 Aceptado el 7 de marzo de 2014.

Este documento se debe citar como: Pérez V., M.A., M.J. González-Guillén, J.R. Valdez-Lazalde, H.M. De los Santos-Posadas y G. Ángeles-Pérez. 2014. Aptitud ecoturística en la sierra nevada de Texcoco, Estado de México. Madera y Bosques 20(2):127-140 